\title{
Laboratory astrophysics: Investigation of planetary and astrophysical maser emission
}

\author{
R. Bingham ${ }^{1,2}$, D.C. Speirs ${ }^{2}$, B.J. Kellett ${ }^{1}$, I. Vorgul $^{3}$, S.L. McConville ${ }^{2}$, \\ R.A. Cairns ${ }^{3}$, A.W. Cross ${ }^{2}$, A.D.R. Phelps ${ }^{2}$ and K. Ronald ${ }^{2}$ \\ ${ }^{1}$ Central Laser Facility, STFC Rutherford Appleton Laboratory, Chilton, OX11 \\ OQX, U.K. \\ ${ }^{2}$ Department of Physics, SUPA, University of Strathclyde, Glasgow, G4 ONG, \\ U.K. \\ ${ }^{3}$ School of Mathematics and Statistics, University of St Andrews, St Andrews, \\ KY16 9SS, U.K.
}

\begin{abstract}
This paper describes a model for cyclotron maser emission applicable to planetary auroral radio emission, the stars UV Ceti and CU Virginus, blazar jets and astrophysical shocks. These emissions may be attributed to energetic electrons moving into convergent magnetic fields that are typically found in association with dipole like planetary magnetospheres or shocks. It is found that magnetic compression leads to the formation of a velocity distribution having a horseshoe shape as a result of conservation of the electron magnetic moment. Under certain plasma conditions where the local electron plasma frequency $\omega_{\mathrm{pe}}$ is much less than the cyclotron frequency $\omega_{\mathrm{ce}}$ the distribution is found to be unstable to maser type radiation emission. We have established a laboratory-based facility that has verified many of the details of our original theoretical description and agrees well with numerical simulations. The experiment has demonstrated that the horseshoe distribution produces cyclotron emission at a frequency just below the local electron cyclotron frequency, with polarization close to X-mode and propagating nearly perpendicularly to the electron beam motion. We discuss recent developments in the theory and simulation of the instability including addressing radiation escape problems, and relate these to the laboratory, space, and astrophysical observations. The experiments showed strong narrow band EM emissions at frequencies just below the cold-plasma cyclotron frequency as predicted by the theory. Measurements of the conversion efficiency, mode and spectral content were in close agreement with the predictions of numerical simulations undertaken using a particle-in-cell code and also with satellite observations confirming the horseshoe maser as an important emission mechanism in geophysical / astrophysical plasmas. In each case we address how the radiation can escape the plasma without suffering strong absorption at the second harmonic layer.
\end{abstract}

Keywords Auroral kilometric radiation; cyclotron maser radiation; plasma instabilities; blazar jets; 


\section{Introduction}

At least five sources of intense, non-thermal planetary radio emission are known in our solar system. The Earth, Jupiter, Saturn, Uranus and Neptune all produce strong maser emission in the kilometre to decametre wavelength range [Zarka, 1992]. In astrophysical environments the cyclotron maser instability is an important mechanism that provides an explanation for observations of intense microwave radiation [Treumann 2006]. Radio emission from stars with a dipole magnetic field UV Ceti [Benz et al.1998, Kellett et al. 2002] and Cu Virginus [Trigilio et al. 2000, Hatzes 1997, Kellett et al. 2007, Lo et al. 2012], blazar jets [Begelman et al. 2005, Treumann 2006] and shocks [Bingham et al. 2003] are also thought to be associated with maser emission. Cyclotron maser emission from astrophysical objects was first investigated by Twiss [Twiss 1958]. The radiation is associated with electron beams accelerated into increasing magnetic fields where the local electron plasma frequency $\omega_{\mathrm{pe}}$ is much less than the cyclotron frequency $\omega_{\text {ce }}$ (see figure 1 for the terrestrial auroral case). In the low density regime, the growth rate of the R-X mode is inversely proportional to the density, while the growth rate of the ordinary mode is proportional to the density. This explains why the RX mode is the dominant feature of radiation in regions where $\omega_{\mathrm{pe}}<<\omega_{\mathrm{ce}}$ [Melrose and Dulk 1982]. In the case of the Earth's auroral radio emission region, the ratio $\omega_{\mathrm{pe}} / \omega_{\mathrm{ce}} \sim 0.01$, for blazar jets this ratio can be even smaller [Begelmann et al. 2005, Treumann 2006]. The idea that planetary radio emission is the result of a maser instability has been current for many years [ $\mathrm{Wu}$ and Lee 1979, Winglee and Pritchett 1986, Melrose 1986, Louarn et al. 1990, Louarn and Le Queau 1996, Delory et al. 1998, Ergun et al. 2000] with several schemes having been proposed. The electron loss-cone distribution [Wu and Lee 1979; Winglee and Pritchett 1986] was suggested as the population inversion required for maser emission. However there is little or no evidence [Delory et al. 1998; Ergun et al. 2000] of the loss -cone distribution in the satellite data to support this assertion and as pointed out by Melrose (Melrose 1998), for the emission to be explained by a loss-cone instability it has to be supposed that all observations are of a saturated state after the loss -cone has filled by pitch angle diffusion. The loss-cone instability was also questioned as the source of terrestrial auroral kilometric radiation (AKR) by the results of particle-in-cell simulations 
conducted by Pritchett [Pritchett, 1986; Pritchett et al. 1999], based on observed electron distribution functions. These simulations show that the loss-cone instability is not a particularly efficient process. Observations from the Viking spacecraft [Louarn et al. 1990; Roux et al. 1993] in the Earth's auroral magnetosphere led to the suggestion that the energy source for AKR is instead a population of downwards accelerated electrons with a large perpendicular velocity produced by a combination of parallel accelerating electric field and converging magnetic field lines. The electron distribution that is formed by beam electrons moving into an increasing magnetic field takes the form of a horseshoe shape. These earlier observations have been backed up by more recent data from the Fast Auroral SnapshoT (FAST) satellite [Delory et al. 1998; Ergun et al. 2000; Pritchett et al. 2002] that clearly demonstrate a strong correlation between the electromagnetic wave emission and the occurrence of the horseshoe distribution. A number of authors [Delory et al. 1998; Ergun et al. 2000; Pritchett et al. 2002; Pritchett et al. 1999; Bingham and Cairns 2000; Bingham et al. 2004; Vorgul et al. 2005; Cairns et al. 2005; Speirs et al. 2005; Ronald et al. 2008; Ronald et al. 2008b; McConville et al. 2008; Speirs et al. 2008; Gillespie et al. 2008] have recently focused on the horseshoe distribution as the main source of AKR and demonstrated a robust cyclotron maser instability with a large spatial growth rate [Bingham and Cairns 2000; Bingham et al. 2004; Vorgul et al 2005], much larger than for a loss-cone distribution.

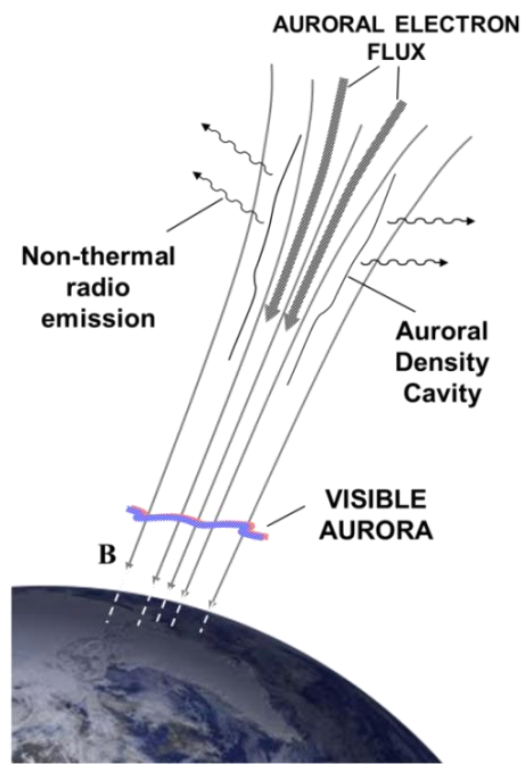

FIG. 1. Diagrammatic representation of the terrestrial auroral process. 
Other characteristics that are associated with the horseshoe maser but not the losscone maser are high brightness temperatures $>10^{14} \mathrm{~K}$, continuous emission and high efficiency of order 1\% [Gurnett 1974]. Given the prevalence of converging magnetic fields in astrophysics it has been suggested that the horseshoe maser can explain radio emission from stars with a dipole magnetic field [Benz et al.1998, Kellett et al. 2002 \& 2007, Trigilio et al. 2000, Lo et al. 2012], blazar jets [Begelman et al. 2005] and shocks [Bingham et al. 2003]. Since the dispersion relation describing the electromagnetic wave only depends on the factor by which the magnetic field increases and on ratios of the plasma and cyclotron frequencies, it is possible to scale the effect to laboratory dimensions. An experiment was designed and built to test the different cyclotron maser instabilities driven by loss cone and horseshoe distributions - a difficult task in the space environment. The experimental apparatus devised consisted of an electron beam injected into the convergent / fringing field of a magnetic solenoid configuration, creating a horseshoe distribution in velocity space similar to those observed by spacecraft [Delory et al. 1998; Ergun et al. 2000; Louarn et al. 1990; Roux et al. 1993; Pritchett 1986] in the auroral region, Figure 2. An important issue for consideration is how the radiation can escape from each of the objects discussed in this paper. For each case we outline possible escape mechanisms.

\section{Observations of cyclotron-maser emission}

\subsection{Satellite observations in the auroral magnetospheric region}

The most convenient source for observation is Earth, and terrestrial AKR has been observed in detail by numerous satellite missions. Such observations from within the source region indicate that AKR is generated at high altitudes $(\sim 1.5 \rightarrow 3$ Earth radii) in cavities of low background plasma density aligned along the auroral magnetic field. From the first images of the terrestrial auroral electron distribution function (DE-1 satellite), a definite crescent, or what we call a horseshoe, can be seen [Menietti and Burch 1985]. Later observations by the Viking and FAST satellites provided much clearer images where one can see an obvious, highly populated horseshoe [Delory et al. 1998; Ergun et al. 2000; Pritchett et al. 2002]. The main results of the satellite observations relevant to cyclotron maser emission are: the existence of fast electrons of approximately the same energy moving into 
a convergent magnetic field, an observed horseshoe shaped electron momentum distribution, intense kilometric radiation nearly perpendicular to the magnetostatic field, a well-defined spectral output at around the electron cyclotron frequency, Xmode polarisation of the emissions within the source region and an estimated emission efficiency of about 1\% [Menietti and Burch 1985; Gurnett 1974; Mutel et al. 2008; Menietti et al. 2011].
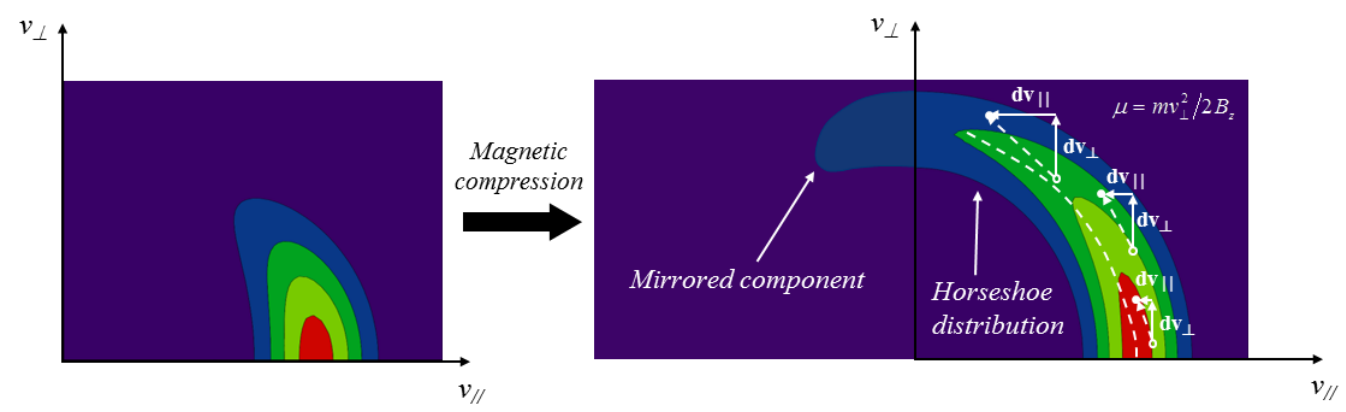

FIG. 2. Illustrative diagram showing the formation of a horseshoe shaped velocity distribution in an electron beam subject to magnetic compression.

An important issue for consideration is how the radiation can escape from the auroral magnetospheric plasma into space. The cyclotron maser mechanism generates radiation propagating almost perpendicular to the magnetic field in the extraordinary mode. Melrose and Dulk 1982 and Melrose 1999 argue that strong absorption will take place when the radiation encounters the second harmonic absorption layer. Second harmonic absorption is maximum when the radiation propagates perpendicular to the magnetic field and goes to zero as $\theta$ approaches $0^{\circ}$, inferring that for radiation propagating at small angles to the magnetic field attenuation is small [Melrose 1986]. Recent work by Mutel et al 2008 using data obtained by the four spacecraft Cluster mission show that the radiation from the auroral region undergoes strong refraction, caused by the density inhomogeneity in the magnetospheric plasma with altitude [Mutel et al. 2008, Menietti et al. 2011]. Mutel et al. have shown that the ray paths due to refraction are propagating at angles with respect to the magnetic field of between $10^{\circ}$ and $20^{\circ}$ well before they encounter the second harmonic layer, with much reduced attenuation at these angles. Both simulations [Speirs et al. 2010] and observations [Menietti et al. 2011, Mutel et al. 2008] suggest that the radiation is generated at a backward angle of a few degrees to the beam direction and not strictly perpendicular. This serves as a suitable precursor to upward refraction with respect to the decreasing 
background plasma density with increasing altitude as observed by Menietti et al. 2011 and Mutel et al. 2008.

\subsection{Stellar observations}

Prompted by the first radio resolved image of the star UV Ceti [Benz et al. 1998], we undertook a review of the $\mathrm{x}$-ray and radio observations of active late-type stars (in general), and UV Ceti, in particular. One of the aspects of the observations that we particularly noted was the highly polarized (nearly $100 \%$ right-hand circular polarised along the direction of propagation) radio outbursts of UV Ceti. We also noted the high degree of correlation observed between the x-ray and radio luminosity of active late-type stars. Taken together, these observations strongly suggested that a new radio emission mechanism was responsible for most (and possibly all) of the radio emission of active late-type stars.

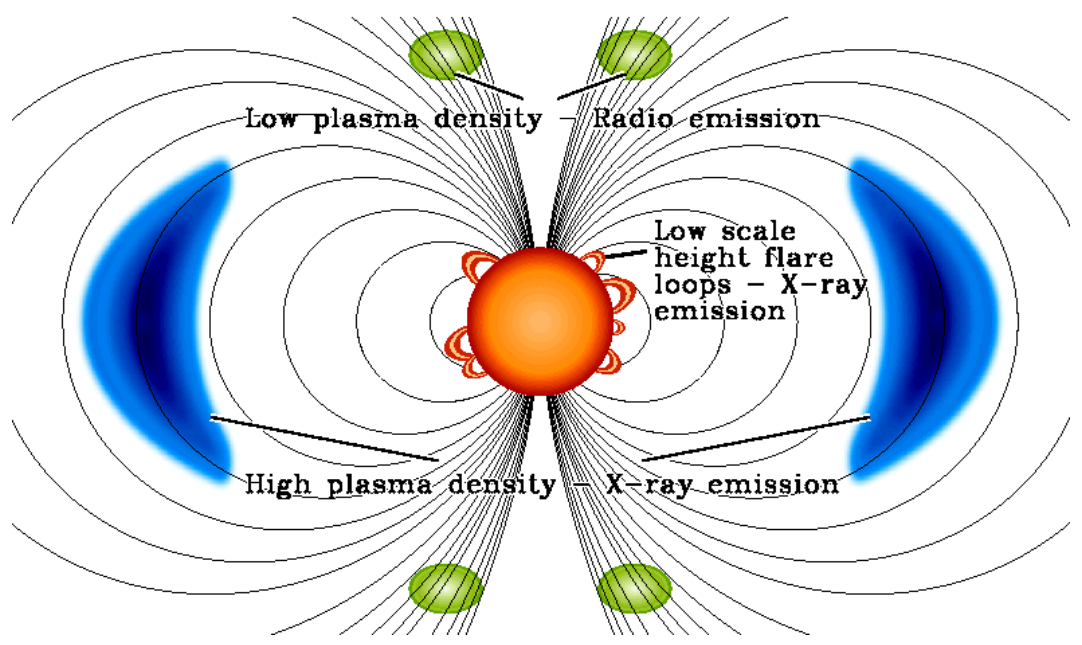

FIG. 3. Diagrammatic representation of radiation emission from the flare star UV-Ceti [Kellett et al. 2002].

The first resolved image from UV Ceti [Benz et al. 1998] showed that the radio emission was emerging from the poles of the star. In the analysis by Benz et al. they estimated that the plasma density was $<10^{8} \mathrm{~cm}^{-3}$ giving a plasma frequency of $<10^{8} \mathrm{~Hz}$, i.e. much less than the observed emission frequency of $8.33 \mathrm{GHz}$. Combined with a typical magnetic flux density of order 1000Gauss [Vogt 1980], we were prompted to develop a cyclotron maser emission model driven by an electron beam entering the polar converging magnetic region of the star [Kellett et al. 2002; Bingham et al. 2001]. The fact that this mechanism could easily generate the observed radio flux from these stars with $\mathrm{keV}$ energy electrons could then also 
help explain the $\mathrm{x}$-ray/radio correlation. X-ray emission also implies keV electrons, so it was possible or even likely that the same electrons were responsible for the x-ray and radio emission - therefore easily explaining the observed correlation [Kellett et al. 2002]. Our model for UV Ceti (Bingham et al. 2001) is summarized in Fig. 3. We adopt a dipole field geometry as suggested by the observations. Similar to the planetary case, a plasma density gradient would refract the radiation out of the plane perpendicular to the magnetic field resulting in propagation close to the magnetic field direction before encountering the second harmonic absorption layer, allowing the radiation to escape with little absorption. A similar dipole model is also invoked for CU Virginis where we see pulsed radiation signatures that very much suggest a continuous radiation source [Kellett et al. 2007, Lo et al. 2012] but which appears pulsed due to the rotation of the source across the Earth's plane. This has been coined by Trigilio the "lighthouse effect" reminiscent of pulsars but which we could call pulstars [Trigilio et al. 2007].

We conclude that maser emission from young active stars with a strong dipolelike magnetic field [Benz et al. 1998, Kellett et al. 2002, Bingham et al. 2001] may be similar to the planetary auroral models in terms of the strong refraction induced by altitude variation in magnetospheric plasma density, allowing the radiation to easily pass through the second harmonic layer with little attenuation. This may not be so for solar radio emission which is associated with a much more complex magnetic field structure than stars like UV-Ceti and CU Virginus which have a strong dipole magnetic field [Benz et al. 1998, Hatzes 1997, Borra and Landstreet 1980]. It is highly likely that these stars do indeed emit cyclotron radiation similar to planets from the polar regions of the dipole that correspond to the auroral regions of planets. In this case we would expect the radiation to be emitted initially close to perpendicular and refract upwards due to the plasma density decreasing with altitude. A plasma emission mechanism cannot be ruled out for stars such as the sun that have more complicated magnetic field structures [Thejappa G. et al. 2012, Hillan et al. 2012], however stars such as UV-Ceti and CU-Virginus rotate much faster and have strong dipole magnetic fields of order 1000Gauss [Benz et al. 1998, Hatzes 1997, Borra and Landstreet 1980, Vogt 1980]. 
Stellar and planetary cyclotron maser emission is a significant source of information on planetary / stellar magnetospheres. For offset magnetic / rotational poles, it can also allow us to study the angular momentum evolution of stars and planets in order to detect variations in rotational period [Pyper et al. 1998, Adelman et al. 2001]. The common features of such astrophysical radio sources at any scale are the presence of a strong dipole or converging magnetic field, the existence of energetic electrons and observed, highly circularly polarised radio emissions at a range of frequencies correlating with the source region range in electron cyclotron frequency [Treumann 2006, Zarka 1992]

\subsection{Blazar jets and shocks}

Relativistic jets (or blazar jets when directed towards the Earth) are beam-like linear features observable over a broad range of frequencies and generated perpendicular to the accretion disc of super massive black holes [Meier et al. 2001, Nemmen et al. 2012]. They can extend over intergalactic distances (many thousands of light years) and have been observed to generate highly non-thermal radio emission at frequencies ranging from a few to 100's of GHz. It has been suggested that a cyclotron-maser instability may be responsible for the generation of these emissions within the low density $\left(\omega_{\mathrm{ce}}>\omega_{\mathrm{pe}}\right)$, magnetised relativistic electron population of the jet [Bingham et al. 2003, Begelman et al. 2005, Treumann et al. 2006]. It is believed that small scale magnetic mirrors / convergent flux tubes may be formed within the jet via hydrodynamic instabilities or shocks, providing the means of generating the required electron velocity distribution [Bingham et al. 2003, Begelman et al. 2005].

The magnetic guide fields within a blazar jet are believed to be very strong [O'Sullivan and Gabuzda 2009] and for the highly energetic electron population within the jet this can lead to large relativistic electron cyclotron frequencies in the 100's of GHz range such that $\omega_{\mathrm{ce}} \gg \omega_{\mathrm{pe}}$ [Begelman et al. 2005]. The values used by Begelman, Ergun and Rees for the ratio of cyclotron frequency to plasma frequency indicate that the efficiency of the emission process for blazar jets could be an order of magnitude greater than for the planetary auroral case. This forms part of the argument used by Begelmann, Ergun and Rees to conclude that the 
radiation could indeed be due to the cyclotron-maser instability driven by an electron horseshoe / ring distribution. Previous work has shown how a suitable ring-type distribution may be generated directly by energisation of particles perpendicular the magnetostatic field via the surfatron mechanism [Katsouleas and Dawson 1983]. We have also previously proposed a scheme by which electrons may be accelerated and subject to magnetic compression within a magnetised collisionless shock, generating a suitable crescent or horseshoe-type velocity distribution [Bingham et al. 2003]. Both such mechanisms are believed to be viable within the highly magnetised, turbulent plasma of a blazar jet, with small-scale magnetic mirrors and field aligned currents expected to occur in association with quasi-perpendicular collisionless shocks [Begelman et al. 2005]. Counterstreaming ion and ring distributions generated at such shocks can excite lower hybrid waves via the modified two-stream instability that are capable of accelerating electrons to high energies, parallel to the magnetic field [McClements et al. 1993]. These high energy tail distributions can then be subject to magnetic compression when moving from the upstream to downstream region of the magnetised collisionless shock, forming a horseshoe-type distribution suitable for driving the cyclotron-maser instability.

Propagation and escape of cyclotron-maser radiation from a blazar jet has been considered in some detail [Begelman et al. 2005, Treumann et al. 2006]. Among the various factors debated, second harmonic cyclotron absorption and synchrotron absorption are potentially the most significant impediments. These can however be suitably accounted for [Begelman et al. 2005] in the case of a blazar jet, where the second harmonic absorption layer is considered to occur transversely with respect to the magnetostatic field [Begelman et al. 2005] . Begelmann et al. 2005 have shown as the generated X-mode radiation propagates radially outwards, where the field can be approximated as dropping off linearly as $\mathrm{B}=\mathrm{B}_{\mathrm{m}} \mathrm{r}_{\mathrm{m}} / \mathrm{r}$ with $\mathrm{B}_{\mathrm{m}}$ and $\mathrm{r}_{\mathrm{m}}$ the magnetic flux density and radius at the maser emission source respectively. Begelman et al. 2005 show that the thickness of the second harmonic layer can be approximated as $\sim \mathrm{r}_{\mathrm{m}} \omega_{\mathrm{pe}} / \omega_{\mathrm{ce}}$ which is relatively thin and results in attenuation of $\sim 10 \%$. Another factor not previously considered is the potential for refraction of the generated radiation due to the plasma density gradient associated with quasi-perpendicular shock itself. Akin to our previous 
consideration for the terrestrial auroral case, this could result in R-mode like radiation propagation and an associated reduction in cyclotron-wave coupling efficiency for second harmonic absorption in a layer parallel to the magnetic field.

\section{Theory and Simulations}

\subsection{Kinetic Analysis}

An analytic form of the electron distribution function with different opening angles, energy spread and density ratios between the hot component making up the horseshoe part and a background Maxwellian component is used in a dispersion relation for the $\mathrm{R}-\mathrm{X}$ mode which is easily obtainable from the susceptibility tensor given by Stix [Stix 1992]. We shall assume that the frequency is close to the electron cyclotron frequency, and also assume that the Larmor radius is much less than the wavelength for typical electron velocities. This latter condition means that we need only consider the susceptibility to lowest order in $\mathrm{k}_{\perp} \mathrm{v}_{\perp} / \Omega_{\mathrm{e}}$. If we neglect all but the zero order terms we get the cold plasma result. To a first approximation we need only take account of the velocity distribution of the electrons in the resonant integral which involves $1 /\left(\omega-\Omega_{\mathrm{e}}\right)$ where $\Omega_{\mathrm{e}}$ is the relativistic electron cyclotron frequency $\mathrm{eB} / \gamma \mathrm{m}_{\mathrm{e}}$ with e the electron charge, $B$ the magnetic field $\gamma$ the Lorenz factor and $m_{e}$ the electron rest mass. In this resonant term we must take account of the relativistic shift of the cyclotron frequency, since this picks out a particular group of resonant electrons and produces damping or growth of the wave. In terms of momentum $\mathrm{p}$ we have

$$
\Omega_{e}=\Omega_{e 0}\left(1+\frac{p^{2}}{m^{2} c^{2}}\right)^{-\frac{1}{2}} \approx \Omega_{e 0}\left(1-\frac{1}{2} \frac{p^{2}}{m^{2} c^{2}}\right)
$$

where $\Omega_{\mathrm{e} 0}$ is the nonrelativistic electron cyclotron frequency. For the real part of the resonant integral we can simply take the cold plasma value. Although this goes as $1 /\left(\omega-\Omega_{\mathrm{e} 0}\right)$ and appears to be near singular at the resonance, the $1 /(\omega-$ $\Omega_{\mathrm{e} 0}$ ) factors in the real part of the dispersion relation cancel out and it behaves quite smoothly in the vicinity of the cyclotron frequency. It is not crucial to include small corrections to the cyclotron frequency in the real part of the dispersion relation. The refractive index $\mathrm{n}$ for the $\mathrm{R}-\mathrm{X}$ mode is given by 


$$
n^{2}=\varepsilon_{\perp}-\frac{\varepsilon_{x y}^{2}}{\varepsilon_{\perp}}
$$

and considering the dielectric tensor elements $\varepsilon_{\perp}$ and $\varepsilon_{\mathrm{xy}}$ with the approximations we have described, an expression for the perturbation in refractive index $\eta$ due to the imaginary component can be obtained [Pritchett et al. 2002].

$$
2 n \delta n \approx-i \alpha \frac{\left(\omega-\Omega_{e 0}\right)^{2}\left(2 \Omega_{e 0}^{2}-\omega_{p}^{2}\right)^{2}}{\omega_{p}^{4} \Omega_{e 0}^{2}}
$$

Where

$$
\alpha=\left.\frac{1}{4} \frac{\omega_{p e}^{2}}{\Omega_{e 0}^{2}} 2 \pi^{2} m^{2} c^{2} \int_{-1}^{1} d \mu\left(1-\mu^{2}\right) p^{2}\left(\frac{\partial f_{e}}{\partial p}-\frac{\mu}{p} \frac{\partial f_{e}}{\partial \mu}\right)\right|_{p=p_{0}}
$$

is represented in spherical polar coordinates $(\mathrm{p}, \mu, \phi)$ and $\mu=\cos \vartheta=p_{/ /} / p$

replaces the usual angle $\theta . p_{0}=m c\left(2\left(\Omega_{e 0}-\omega\right) / \Omega_{e 0}\right)^{1 / 2}$ is the resonant momentum.

Substituting the general form of the horseshoe distribution function $f_{0}(p, \mu)=F(p)$ $\mathrm{g}(\mu)$ into $\alpha$ gives

$$
\alpha=\left.\frac{1}{4} \frac{\omega_{p}^{2}}{\Omega_{e 0}^{2}} 2 \pi^{2} m^{2} c^{2} p^{2}\left(P \frac{\partial F}{\partial p}+Q \frac{F}{p}\right)\right|_{p=p_{0}} \text { where } \quad \begin{aligned}
& P=\int_{-1}^{1}\left(1-\mu^{2}\right) g(\mu) d \mu \\
& \text { (1) } \quad \text { (2) }
\end{aligned}=\int_{-1}^{1}\left(1-3 \mu^{2}\right) g(\mu) d \mu
$$

Term (1) is destabilizing, resulting in emission of waves if the gradient of $\mathrm{F}$ is positive at the resonant momentum. The second term is negative if $\mathrm{g}$ is strongly localized around $\mu=1$ and goes to zero if $\mathrm{g}$ becomes uniform on the interval $[-1,1]$. We might, therefore, have a scenario in which a beam moving down the field line with small perpendicular spread is stable. As it moves into a region of higher magnetic field and spreads into a wider horseshoe it can then become unstable and trigger the emission of AKR.

To illustrate the behaviour we consider the case of a horseshoe distribution centred at $\mathrm{p}_{\|}=0.1 \mathrm{~m}_{\mathrm{e}} \mathrm{c}$, typical of the primary auroral electrons, moving down the field lines within a lower density Maxwellian plasma with a thermal temperature of $312 \mathrm{eV}$. Some results suggest that the background Maxwellian may even be 
absent giving rise to the auroral density cavity. The absence of a low density background Maxwellian distribution does not change the characteristics of the instability, in fact it promotes it. We solve Eq. 3 to obtain the perpendicular spatial growth rate $\gamma_{\perp}$ shown in Fig. 4. A typical convective growth length across the magnetic field $\mathrm{L}_{\mathrm{c}}=2 \pi / I m \mathrm{k}_{\perp}$ is $10 \lambda$ for $\mu_{0}=0.5$, thermal width $=0.02 \mathrm{~m}_{\mathrm{e}} \mathrm{c}$ and $\mathrm{n}_{\mathrm{h}}=0.66 \mathrm{n}_{\mathrm{e}}$ and $6 \lambda$ for $\mu_{0}=0.75$, thermal width $=0.05 \mathrm{~m}_{\mathrm{e}} \mathrm{c}$ and $\mathrm{n}_{\mathrm{h}}=0.9 \mathrm{n}_{\mathrm{e}}$ both for a cyclotron frequency of $440 \mathrm{kHz}$. These convective growth distances are $6.8 \mathrm{~km}$ and $4 \mathrm{~km}$, respectively, allowing for many e-foldings within the auroral density cavity, which has a latitudinal width of about $100 \mathrm{~km}$ [Strangeway et al. 1998]. We find that the growth rate decreases for increasing $\mu_{0}$ and increasing thermal width of the horseshoe distribution and increasing density. The bandwidth is also extremely narrow, estimated from Fig. 4 to be of order $0.5 \%$ or around $200 \mathrm{~Hz}$, also in agreement with observations.

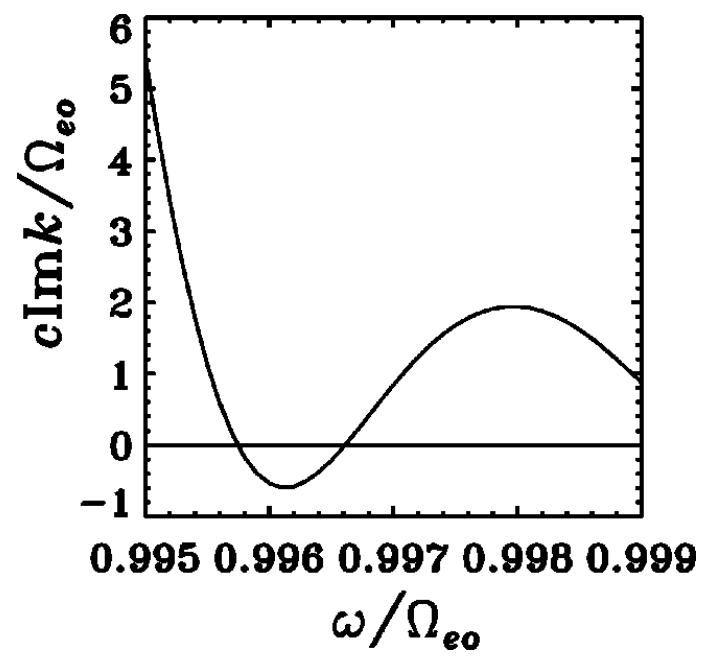

FIG. 4. Spatial growth rate $\operatorname{Im} \mathrm{k}_{\perp}=\gamma_{\perp}$ normalized to $\Omega_{\mathrm{e} 0} / \mathrm{c}$ as a function of frequency.

In a previous analysis we considered the problem of a ring distribution, unstable to a cyclotron maser instability, in a plasma with a magnetic field gradient [Cairns et al. 2008]. This is reminiscent of electron distributions found at shocks and blazar jets [Bingham et al. 2003, Begelman et al. 2005]. We have shown that it is possible to have a localized unstable region around the cyclotron resonance with waves radiating outwards from the beam. 


\subsection{PiC simulations}

For the purpose of simulating the horseshoe distribution formation and any subsequent electromagnetic interaction, the 2D axisymmetric version of the finitedifference time domain PiC (Particle-in-cell) code KARAT was used. KARAT represents the electric and magnetic fields in a simulated geometry as a summation of a static component and a time-varying component [Tarakanov 1992]. The time varying electromagnetic fields generated by charges and currents within the simulation are governed by Maxwell's equations (specifically, Ampere's law and Faraday's law). The motion of PiC particles within these electromagnetic fields are governed by the relativistic Lorentz force equation, with the $\mathrm{PiC}$ particle current density at any point within the simulation geometry determined by the PiC method [Birdsall and Langdon 1985]. The 2D axisymmetric version of KARAT in particular allows indirect observation of the distribution of particles in the transverse plane of motion. The code calculates the $\mathrm{PiC}$ electrons radial and azimuthal velocities and plots each particle location in transverse velocity space. Particles moving in circular orbits transition from $v_{\theta}$ and $v_{r}$ to $-v_{\theta}$ and $-v_{r}$ periodically. The formation of spatial bunches in a cyclotron instability corresponds to modulation of electron gyrational velocities $\left(\mathrm{v}_{\perp}\right)$ and in the limit of small modulation can be perceived in the 2D plots of $\mathrm{v}_{\theta} \mathrm{vs}_{\mathrm{r}}$ as density variations. This is due to the bunch forming in a specific rotational phase with respect to the local $\mathrm{AC}$ field and thus at some instant in time having a "location" in a plot of $\mathrm{v}_{\theta} \mathrm{vs}_{\mathrm{r}}$. In addition, energy modulation can be perceived through changes in the magnitude of $v_{\perp}=\sqrt{v_{\vartheta}^{2}+v_{r}^{2}}$, illustrated by the area spanned by PiC particles in plots of $\mathrm{v}_{\theta} \mathrm{vs}_{\mathrm{r}}$. A PiC simulation was conducted of unbounded electron cyclotron-maser emission. The parameters of this simulation included an axial length (beam propagation path) of $36 \lambda_{\text {ce }}$ (where $\lambda_{\text {ce }}$ is the electron cyclotron free-space wavelength) transverse (radial) dimensions of $5 \lambda_{\text {ce }}$ and radiation absorbent boundaries at the radial and axial limits. A grid resolution of 10 divisions per wavelength at the second harmonic of the electron cyclotron frequency was used along with a PiC merging factor of $3 \times 10^{6}$ electrons / $\mathrm{PiC}$ particle. Figure 5 contains a diagrammatic overview with electron beam trajectory of the unbounded simulation geometry defined within the PiC code KARAT. The electron beam was injected into the simulation with a predefined horseshoe 
distribution, comprising a pitch spread $\alpha=v_{\perp} / v_{\|}$of $0 \rightarrow 9.5$, beam energy of $20 \mathrm{keV} \pm 5 \%$ and beam current of $14 \mathrm{~A}$. A uniform axial magnetic flux density of $0.1 \mathrm{~T}$ was also used in the unbounded simulation as no magnetic compression was necessary.

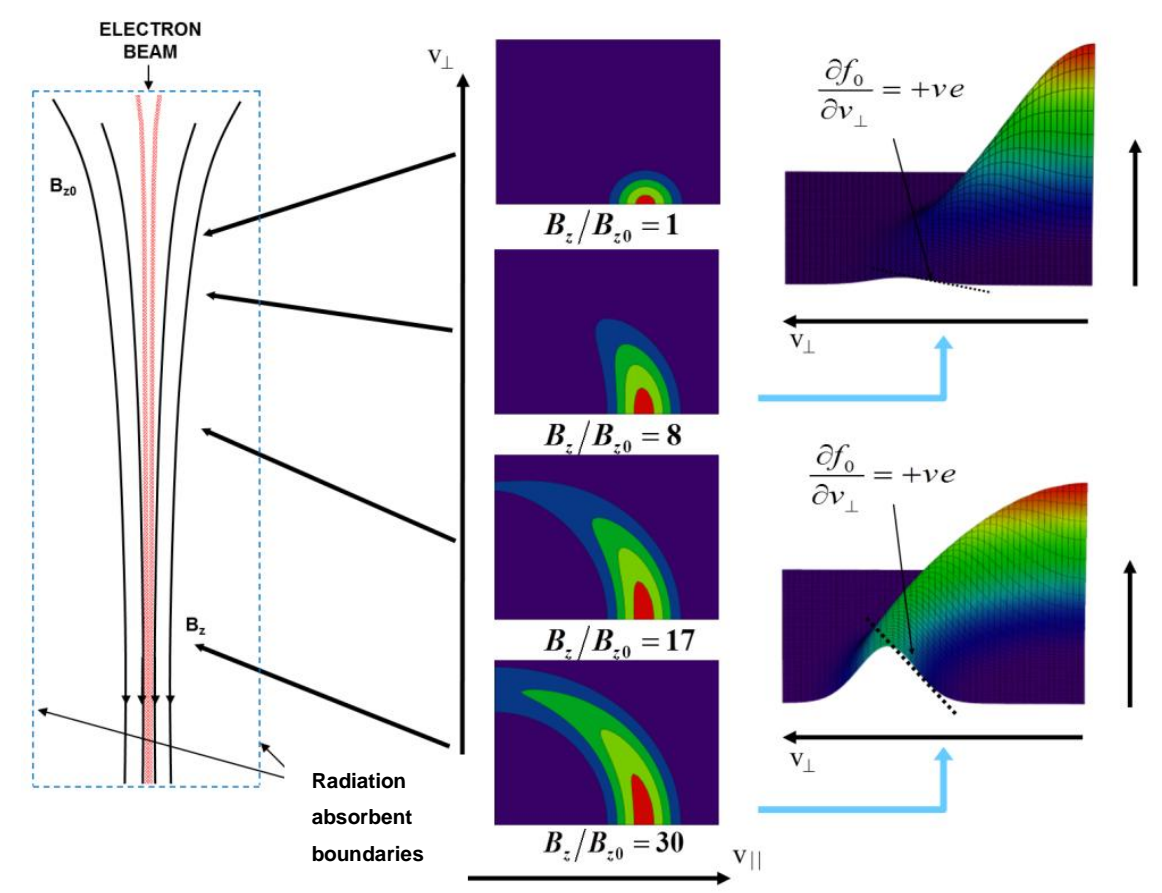

FIG. 5. Diagramatic representation of the PiC simulation geometry, with electromagnetically absorbent boundaries representative of free space.

PiC particle velocity distributions were plotted after a 200ns run time at two axial positions within the simulation geometry. The corresponding data is presented in figure 6 . The injected beam distribution at $\mathrm{z}=0.36 \lambda_{\text {ce }}$ shows a well-defined pitch spread in the $\mathrm{v}_{\perp} \mathrm{VS} \mathrm{v}_{\mathrm{z}}$ plot covering the complete pitch range from an axial electron beam to the point of magnetic mirroring (zero axial velocity). The corresponding $\mathrm{v}_{\theta} \mathrm{vS} \mathrm{v}_{\mathrm{r}}$ plot also shows a uniform spread in relative orbital phase with no evidence of coherent bunching effects. At $z=11.7 \lambda_{\text {ce }}$ the picture is very different however, with clear evidence of azimuthal bunching in the $\mathrm{v}_{\theta} \mathrm{vs}_{\mathrm{r}}$ plot [Chu 2004] and in the corresponding $\mathrm{v}_{\perp}$ vs $\mathrm{v}_{\mathrm{z}}$ plot there is spreading in the transverse velocity profile of high pitch factor electrons. Looking at $\mathrm{z}=18 \lambda_{\text {ce }}$, evidence of phase trapping is now present with a concentration of $\mathrm{PiC}$ particles extending to the origin of the $\mathrm{v}_{\theta} \mathrm{vs} \mathrm{v}_{\mathrm{r}}$ plot. The corresponding $\mathrm{v}_{\perp} \mathrm{vs}_{\mathrm{z}} \mathrm{v}_{\mathrm{z}}$ plot shows a saturated state, with minimum $\partial f / \partial v_{\perp}$. All of these characteristics are strongly 
indicative of resonant particle-wave energy transfer via a cyclotron-maser instability.

Electron (PiC particle) velocity phase space @ $z=0.36 \lambda_{c e}$
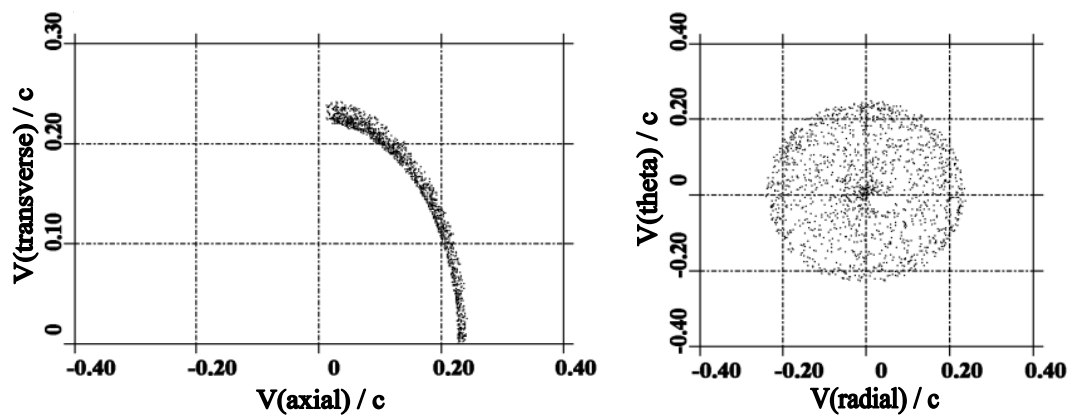

(a)
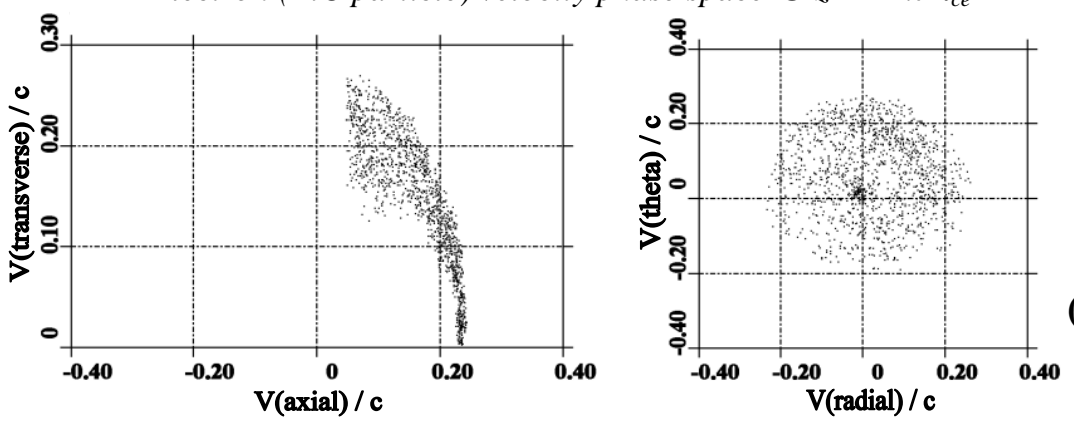

(b)
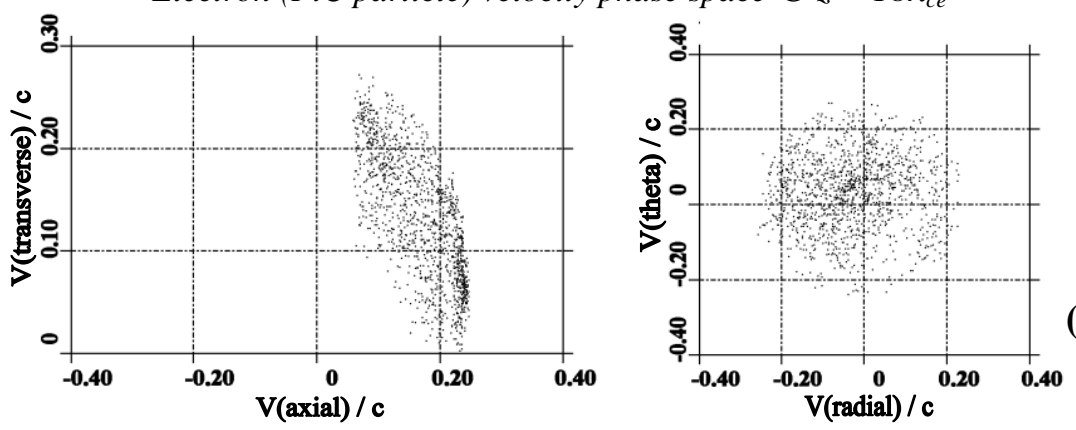

(c)

FIG. 6. PiC particle velocity distributions measured on transverse planes within the simulation geometry at (a) $z=0.36 \lambda_{\text {ce }}$ (b) $z=11.7 \lambda_{\text {ce }}$ and (c) $z=18 \lambda_{\text {ce. }}$

Figure 7 a contains a plot of the radial Poynting flux measured in a plane at $r=$ $0.32 \lambda_{\text {ce }}$, over the entire length of the simulation geometry. A DC offset is present in the measurement due to low frequency electromagnetic field components associated with the electron beam propagation. The rf output power may therefore be obtained from the amplitude of the AC signal superimposed on this DC offset. Looking at figure $7 \mathrm{a}$, there is rather sporadic growth in the electromagnetic output from $54 \mathrm{t} / \mathrm{t}_{\mathrm{ce}}$ onwards (where $\mathrm{t}_{\mathrm{ce}}$ is the electron cyclotron period), with an increased steady growth observed after $432 \mathrm{t} / \mathrm{t}_{\mathrm{ce}}$ saturating at $\sim 2.7 \mathrm{~kW}$ and corresponding to an $\mathrm{rf}$ conversion efficiency of $0.96 \%$. This is comparable to the $1.3 \%$ efficiency 
obtained from earlier waveguide bounded simulations [Speirs et al. 2008] and consistent with the generally accepted estimate of $\sim 1 \%$ for the AKR generation efficiency [Gurnett 1974, Pritchett and Strangeway 1985]. The corresponding output spectra is presented in figure $7 \mathrm{~b}$, showing a well-defined spectral component at $2.68 \mathrm{GHz}$. This represents a $1.1 \%$ downshift from the relativistic electron cyclotron frequency of $2.71 \mathrm{GHz}$.

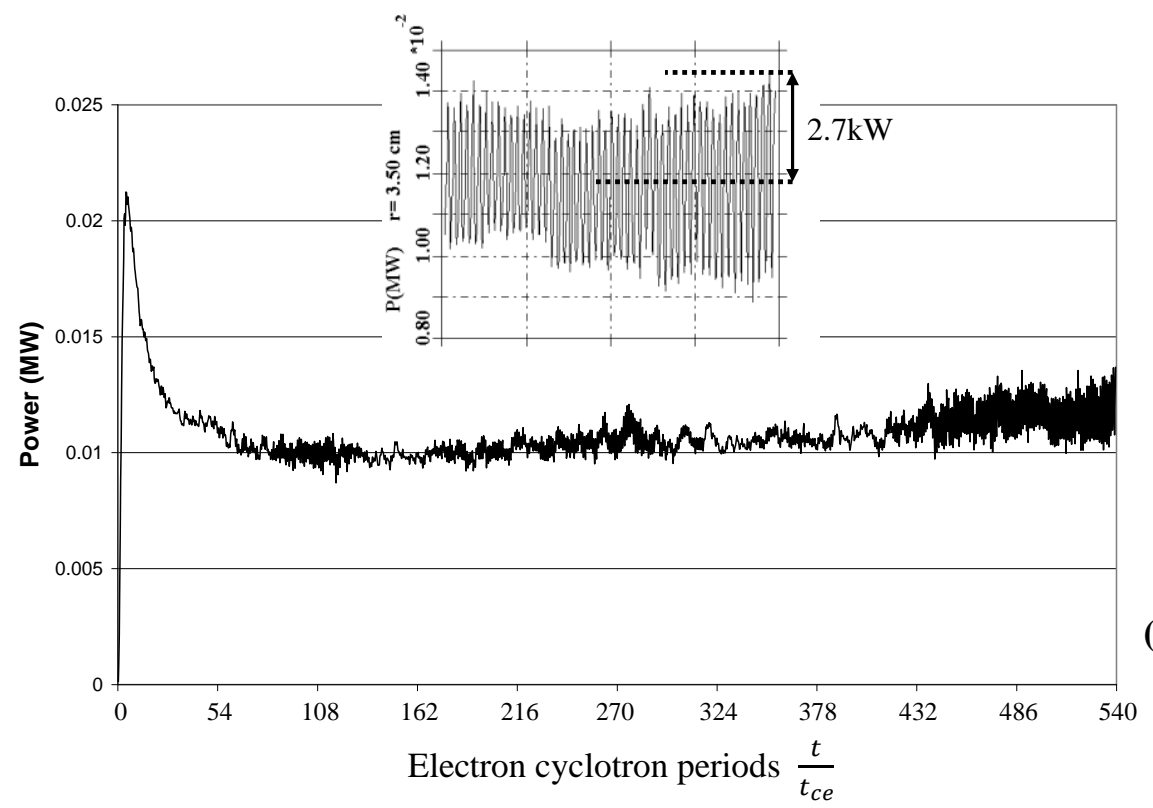

(a)

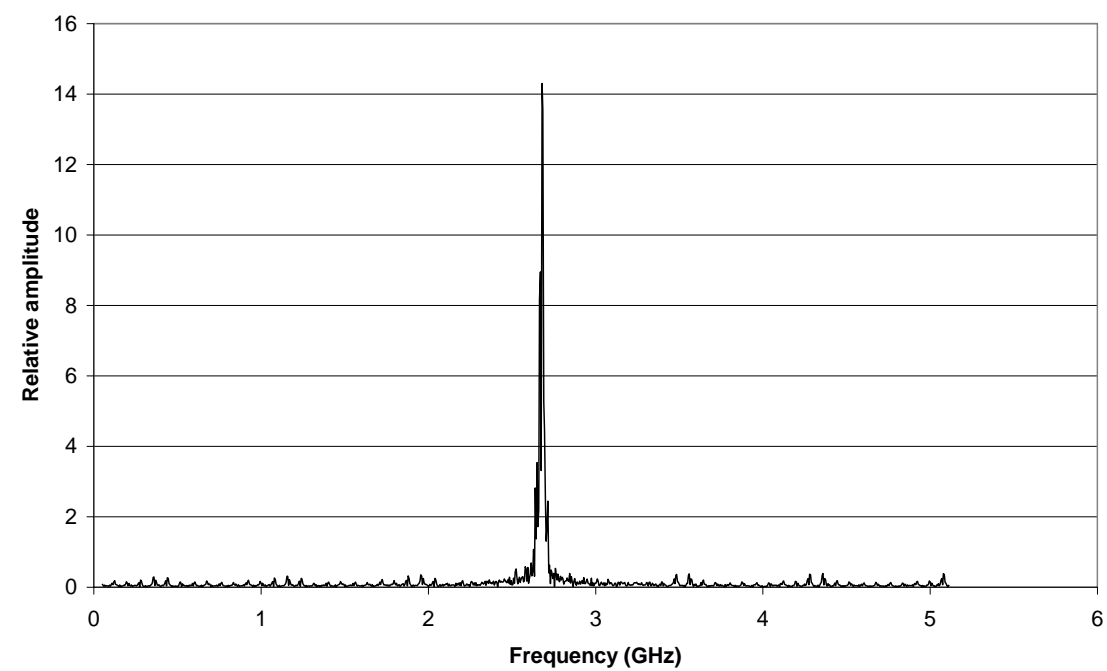

(b)

FIG. 7. (a) Temporal evolution of the radial Poynting flux measured in a plane at $r=0.32 \lambda_{\text {ce }}$ spanning the length of the simulation. (b) Fourier transform of $\mathrm{E}_{\text {theta }}$ from $\mathrm{t} / \mathrm{t}_{\mathrm{ce}}=0 \rightarrow 540$ at $\mathrm{z}=$ $17 \lambda_{\text {ce }}$.

Figure 8 contains a 3D contour plot of $\mathrm{E}_{\text {theta }}$ mapped over the simulation geometry after a 200 ns run time. An electromagnetic wave sourced at $\mathrm{z} \sim 13.5 \lambda_{\text {ce }}$ is evident propagating near perpendicular to the electron beam with a slight backward wave 
character - consistent with the observed $1.1 \%$ downshift in the spectral output. The axial coordinate range over which the wave is generated corresponds to the position at which a spreading in the PiC particle velocity distribution is first observed in figure 6, and represents a significant number of Larmour steps from the point of beam injection for efficient cyclotron-wave coupling to be observed. This represents a reduction in spatial growth rate compared with earlier waveguide-bounded simulations [Speirs et al. 2008], although a comparable saturated RF conversion efficiency was obtained.

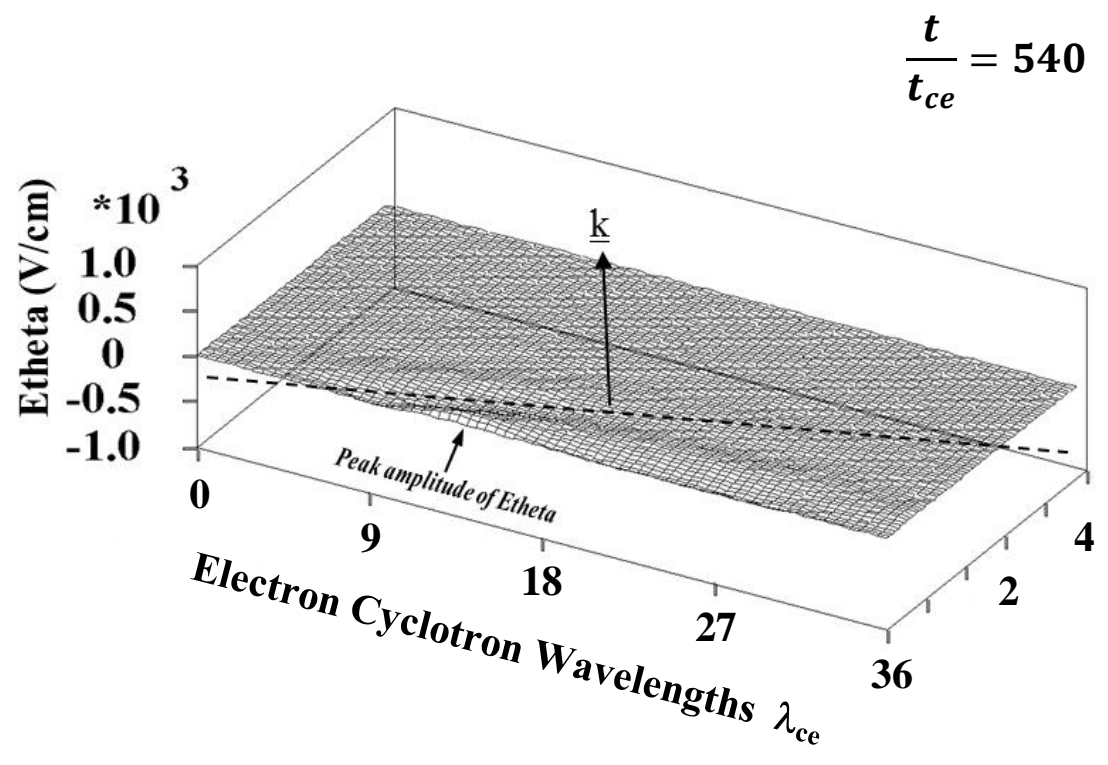

FIG. 8. 3D contour plot of $\mathrm{E}_{\text {theta }}$ within the simulation geometry. RF emission is evident centred around an axial position of $18 \lambda_{\text {ce }}$ and appears to have a slight backward wave character.

\section{Laboratory Experiment}

An experiment was conducted at the University of Strathclyde's beam-plasma laboratory [Speirs et al. 2005; Ronald et al. 2008; Ronald et al. 2008b; McConville et al. 2008] to simulate the magnetic compression of an electron beam and subsequent evolution / stability of the associated electron velocity distribution. The experimental apparatus was based on the use of an electron gun to inject particles into an increasing magnetic field produced by a system of electromagnets. The overall layout of the experiment is illustrated in figure 9 , highlighting the electromagnet configuration formed by six distinct coils. Each coil was wound from OFHC copper tubing coated with a thin plastic sheath for 
electrical insulation. The windings were core cooled by water and were able to carry a current of up to 450A. The first coil (solenoid 1) was half a metre in length and formed of two layers, surrounding the electron accelerator and defining the magnetic flux density experienced by the electrons as they were injected into the $16 \mathrm{~cm}$ diameter beam tunnel. Solenoid 2, also half a metre in length and formed of four layers, confined and transported the electrons to the interaction region and acted as a transition between the low-field electron gun region and the high-field interaction region. As the electron beam diameter reduced with increased flux density, it was possible to reduce the diameter of the interaction region to $8 \mathrm{~cm}$, which also provided a useful reduction in power consumption of the DC solenoid arrangement. Solenoids 3-5 provide the maximum plateau flux density (up to 0.5 $\mathrm{T}$ ) in the apparatus, and were wound as a 10 layer main coil with a pair of two layer balancing shim coils, respectively. Precise control of the magnetic flux density in this 'interaction' region allowed the efficiency of the cyclotron instability to be investigated as a function of cyclotron resonant detuning.

Electron emission into the apparatus was achieved by placing a cathode electrode covered with an annular region ( $3 \mathrm{~cm}$ mean radius, $1 \mathrm{~cm}$ radial width) of velvet emitter [Denisov et al. 1998; Speirs et al. 2005; Ronald et al. 2008] within $2 \mathrm{~cm}$ of a sparse mesh anode, figure 9. Design of this injector was undertaken with the aid of the 2D PiC code KARAT [Speirs et al 2005, 2008]. Application of an accelerating potential of $75 \mathrm{kV}$ to the cathode led to field-enhanced electron emission at the discontinuities of the dielectric fibres. The field emission current was sustained by a tunnelling current and enhanced by an avalanche process within the valance electrons of the fibres and a surface flashover avalanche along the outside of the fibres [Noer 1982; Xu and Latham 1992]. A high current density was emitted from each fibre tip leading to explosive vaporization of the underlying bulk material resulting in the formation of a cathode plasma flare [Mesyats 1991] travelling at a velocity of $\sim 2 \mathrm{~cm} \mu \mathrm{s}^{-1}$ and supporting the spacecharge-limited emission of electrons into the diode gap forming a vacuum spark discharge.

Most of the current traversed the anode mesh and entered the $16 \mathrm{~cm}$ anode can region of the experiment, propagating into the increasing magnetic field. The 
electron gun was placed in the fringing field of solenoid 1, figure 9. As the emission surface was normal to the axis of the solenoids this ensured a variation in the magnitude of magnetic compression as a function of radial position on the annular emitter. This induced pitch variation in combination with a $12^{\circ}$ domed electrode in the centre of the emitter annulus ensured the electrons were emitted with a finite mean pitch factor and pitch spread sufficient to ensure the formation of a horseshoe shaped velocity distribution when subject to significant magnetic compression.

The resultant velocity distribution obtained in the experiment had a population inversion in the perpendicular direction and so would be expected to be unstable to cyclotron-maser emission, since cyclotron resonance produces diffusion predominantly in the perpendicular direction in momentum space. The electron cyclotron resonance condition was defined at the fundamental frequency, with which we shall be concerned here,

$$
\omega=\frac{\omega_{c e}}{\gamma}+k_{\|} v_{\|}
$$

Here $\omega_{\mathrm{ce}}$ is the electron cyclotron frequency, calculated using the rest mass, and $\gamma$ is the usual Lorentz factor. If we consider propagation almost perpendicular to the steady magnetic field, so that the contribution from the Doppler shift is negligible, the locus of resonant particles in $\left(\mathrm{p}_{\perp}, \mathrm{p}_{\|}\right)$is then just a circle in velocity space. If this circle lies around the inside of the horseshoe, then we may expect instability and thus a radiation frequency just below $\omega_{\text {cee }}$. 


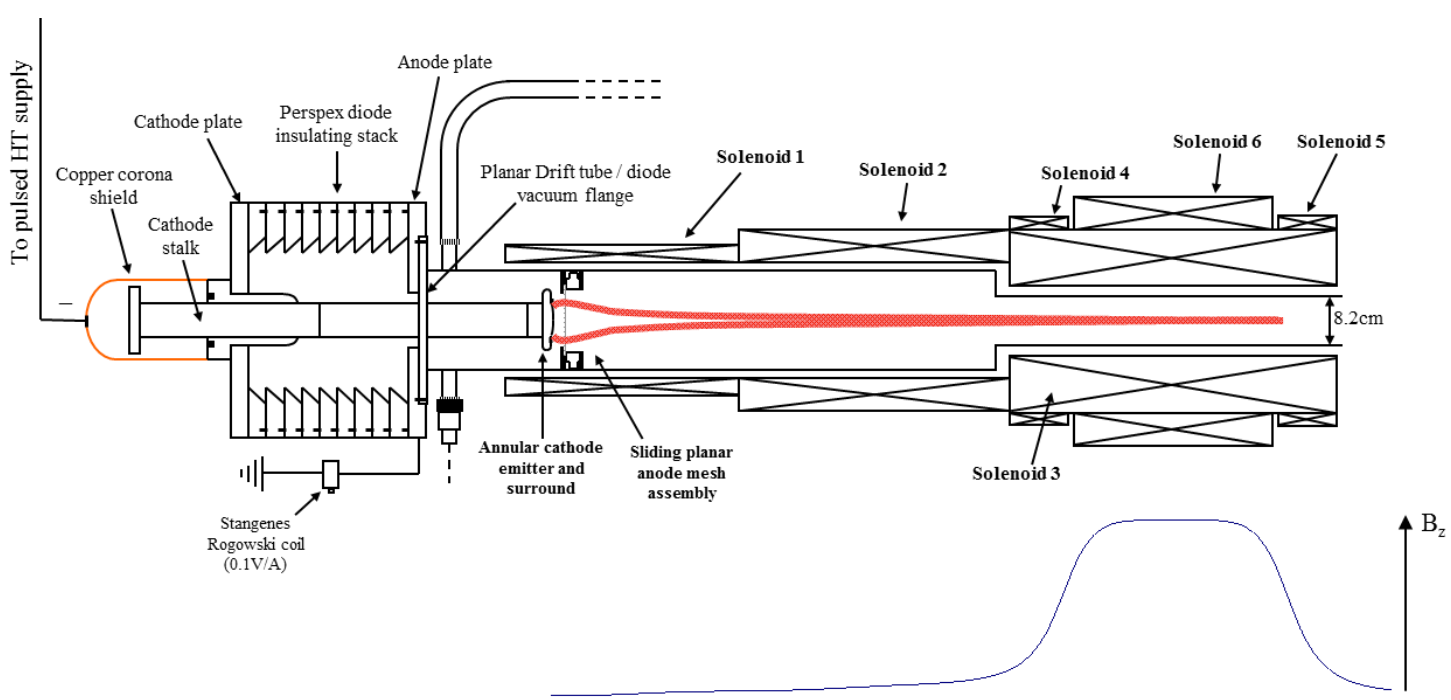

FIG. 9. Schematic diagram of the experimental setup highlighting the magnetic coil configuration and convergent axial magnetic field with peak-plateau region for cyclotron resonant energy transfer.

Near cut-off TE modes were investigated within the interaction waveguide, as these most closely replicate the propagation $(\perp$ static B) and polarisation $(\perp$ static B) properties of the X-mode, minimising the Doppler broadening of the resonance [Speirs et al. 2005, Ronald et al. 2008]. Two interaction regimes were investigated: one comprising a peak (plateau) axial magnetic flux density of $0.18 \mathrm{~T}$ for resonance with the $\mathrm{TE}_{0,1}$ mode, and a second with a peak (plateau) axial magnetic flux density of $0.48 \mathrm{~T}$ for resonance with the $\mathrm{TE}_{0,3}$ mode. The spectra illustrated in Figures 10a and 11a were obtained by Fourier transform of a directly measured $\mathrm{AC}$ waveform acquired by a $12 \mathrm{GHz}$ single-sequence, digital oscilloscope. The first (lower) spectral peak in Figure 10a corresponds to the $\mathrm{TE}_{0,1}$ mode being excited at $4.42 \mathrm{GHz}$, whilst the second harmonic may also be observed at $8.9 \mathrm{GHz}$. The corresponding spectral output for the $11.7 \mathrm{GHz}$ resonance regime is presented in Figure 11a, with a well-defined spectral peak present at $11.7 \mathrm{GHz}$ corresponding to a near cut-off resonance with the $\mathrm{TE}_{0,3}$ mode. On closer inspection some minor splitting is present in the spectral peak, indicative of some slight mode competition with the $\mathrm{TE}_{2,3}$ mode.

Mode coupling within the experiment was diagnosed by scanning single mode rectangular waveguide receivers in the output antenna pattern of the circular interaction waveguide. Both the $4.42 \mathrm{GHz}$ and $11.7 \mathrm{GHz}$ interaction regimes were studied, with figure $10 \mathrm{~b}$ showing the azimuthally polarised antenna pattern 
measured for the $4.42 \mathrm{GHz}$ resonance experiments. The single cycle pattern observed, peaking at $\sim 35$ degrees is indicative of the $\mathrm{TE}_{0,1}$ mode, and consistent with expectations from the beam-wave dispersion characteristics for the experimental parameters [Ronald et al. 2008; Speirs et al. 2008]. Integration over the radial and azimuthal antenna patterns yielded a total output power of $19 \mathrm{~kW}$ corresponding to a beam-wave conversion efficiency of $2 \%$ at optimum cyclotron detuning and a compression ratio of 18 . The compression ratio of 9 yielded $35 \mathrm{~kW}$ and $1 \%$ efficiency, consistent with expectations of the lower compression ratio yielding a reduction in electron population density at higher pitch factors.

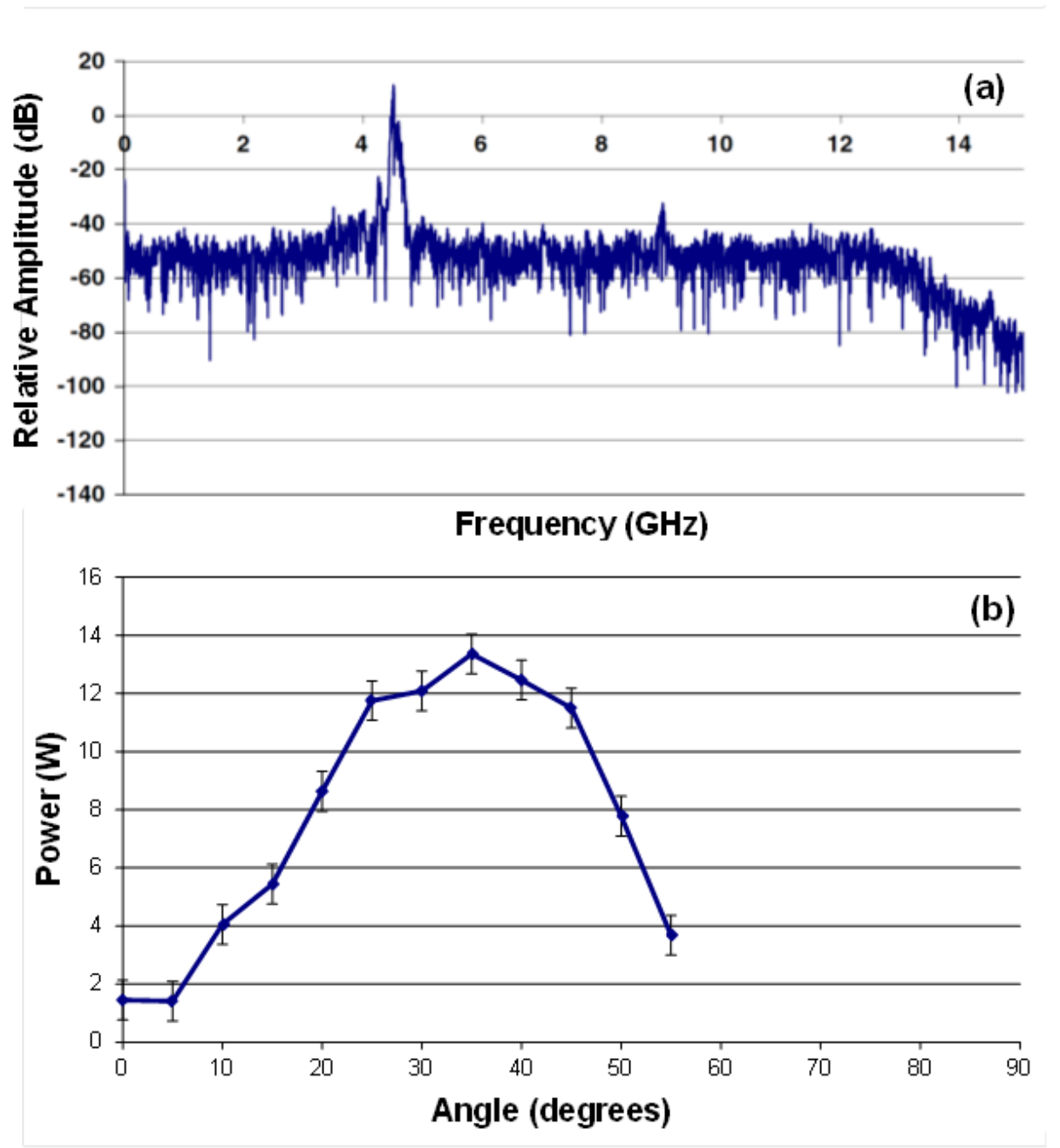

FIG. 10. Experimental measurements for the $4.42 \mathrm{GHz}$ resonance, illustrating (a) the spectrum of the output signal, displaying a strong resonance close to the electron cyclotron frequency, $4.42 \mathrm{GHz}$ and (b) the antenna pattern confirming excitation of the near cut-off $\mathrm{TE}_{0,1}$ mode and yielding the efficiency by integration.

For the $11.7 \mathrm{GHz}$ resonance regime, multimode excitation was observed with some evidence of the $\mathrm{TE}_{2,3}$ mode. The emission was also bursty, with two distinct 
temporal peaks in the output pulse envelope. Figure $11 \mathrm{~b}$ shows the corresponding azimuthal antenna pattern for each of these temporal peaks at a magnetic compression ratio of 16 . The triple peak structure observed is characteristic of both the $\mathrm{TE}_{0,3}$ and $\mathrm{TE}_{2,3}$ modes. Integrating over the corresponding radial and azimuthal antenna patterns yielded an output power of $30 \mathrm{~kW}$ and beam-wave conversion efficiency of $\sim 1 \%$. Overall, the efficiencies obtained for both the $\mathrm{TE}_{0,1}$ and $\mathrm{TE}_{0,3}$ regimes were in close agreement with the predictions of numerical simulations and are consistent with efficiency estimates for the generation of AKR [Gurnett 1974].
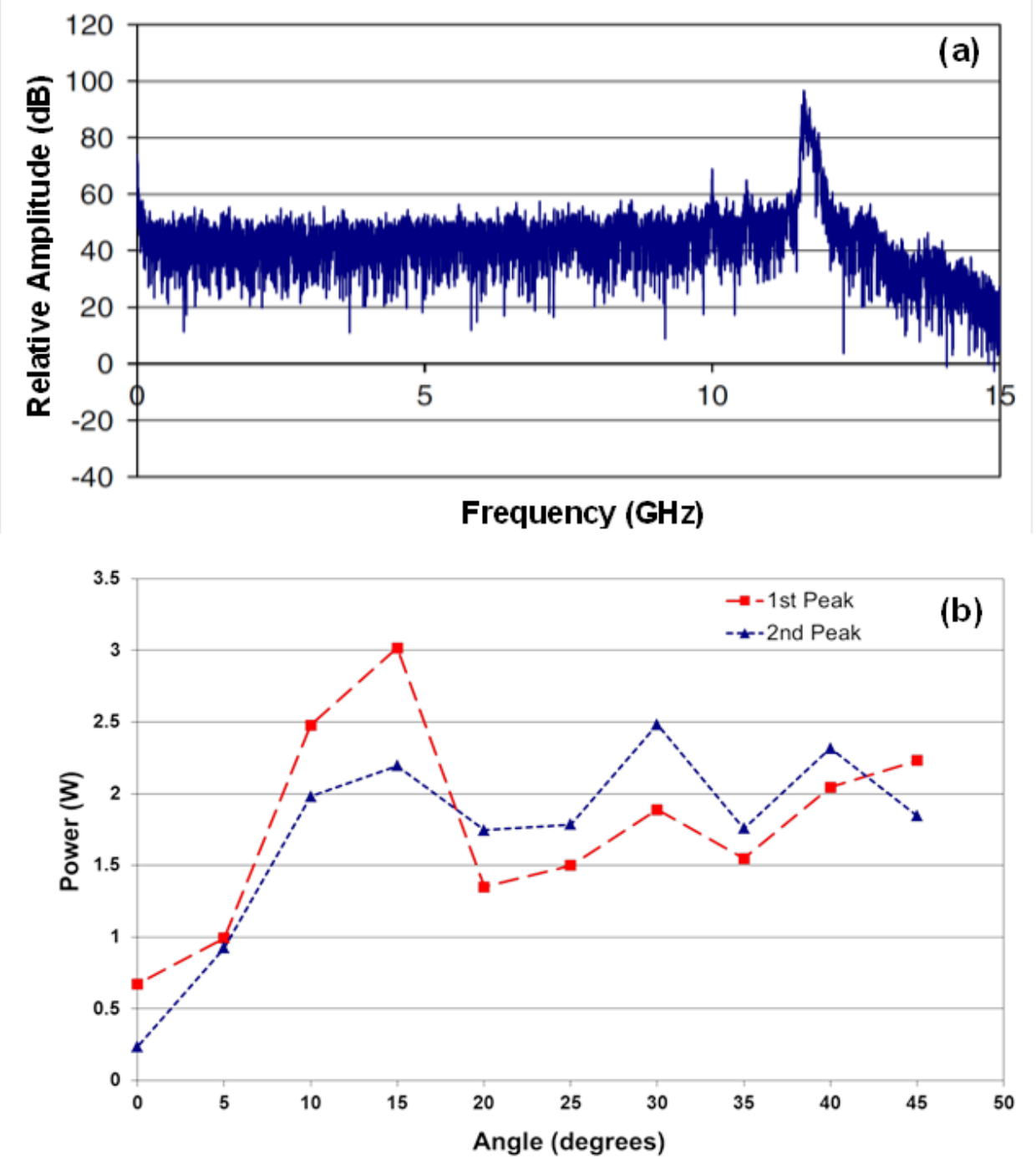

FIG. 11. Experimental measurements for the $11.7 \mathrm{GHz}$ resonance, illustrating (a) the spectrum of the output signal, displaying a strong resonance close to the electron cyclotron frequency, $11.7 \mathrm{GHz}$ and $(\mathrm{b})$ the antenna patterns confirming excitation of the near cut-off $\mathrm{TE}_{0,3}$ mode and yielding the efficiency by integration. 


\section{Conclusions}

This paper describes an experiment created to reproduce an electron distribution function similar to those observed to be correlated with the phenomenon of cyclotron maser radiation commonly found in space and astrophysical plasmas such as planetary aurora, young active stars with a dipole-like magnetic field, blazar jets and shocks. The experiment successfully produced the required electron velocity space distribution. The radiation is associated with electron beams accelerated downwards into the increasing magnetic dipole field of the auroral regions where the local electron plasma frequency $\omega_{\mathrm{pe}}$ is much less than the cyclotron frequency $\omega_{\text {ce }}$. Magnetic compression leads to the formation of a velocity distribution having a horseshoe shape as a result of conservation of magnetic moment. Good agreement on both the efficiency and spectrum of the emitted radiation were obtained between experiments and numerical simulations at 4.45 and $11.7 \mathrm{GHz}$, just below $\omega_{\text {ce. }}$. The evolution of the electron velocity distribution predicted by the simulations and the narrow bandwidth of the radiation emissions appears consistent with the expectation of the theoretical models. Numerical and experimental efficiencies are comparable with the satellite observations of auroral kilometric radiation. The maser radiation is initially beamed perpendicular to the magnetic field - an important factor that has bearing on how the radiation can escape. This has been shown to be possible in the planetary case by the introduction of a plasma density profile in altitude that refracts the radiation sufficiently resulting in the beam propagating with small angles to the magnetic field, reducing the attenuation at the second harmonic layer [Mutel et al 2008]. We adopted a similar model for the stars UV Ceti and CU Virginus which both have strong dipole fields. In the case of blaser jets, Begelman et al. 2005 have demonstrated that second harmonic absorption should be low and the radiation can escape if the source region is close to the boundary in a sufficiently thin layer.

Acknowledgements This work was supported by the EPSRC and the STFC Centre for Fundamental Physics. The authors would like to thank W. He, D. Melrose and V.L. Tarakanov for helpful discussions. 


\section{References}

Adelman, S.K. et al., "On the rotation of the chemically peculiar magnetic star 56 Arietis", Astron. Astrophys., 375, 982-988 (2001).

Begelman M.C, Ergun R.E., Rees M.J., ”Cyclotron maser emission from blazar jets?”, ApJ 625, 51-59 (2005).

Benz A.O. et al., "First VLBI images of a main-sequence star", Astron. Astrophys. 331, 596 (1998).

Bingham R., Cairns R.A., "Generation of auroral kilometric radiation by electron horseshoe distributions", Phys. Plasmas 7, 3089-3092 (2000).

Bingham R, Cairns R.A., Kellett B.J., “Coherent cyclotron maser radiation from UV Ceti”, Astron. Astrophys. 370, 1000-1003 (2001).

Bingham R., Kellett B.J., Cairns R.A., Tonge J., Mendonca J.T., "Cyclotron maser radiation from astrophysical shocks", Astrophysical Journal 595, 279-284 (2003).

Bingham, R., Kellett, B.J., Cairns, R.A. et al., "Cyclotron Maser Radiation in Space and Laboratory Plasmas”, Contrib. Plasma Physics 44, 382-387 (2004).

Birdsall C.K., Langdon A.B., "Plasma Physics Via Computer Simulation”, McGraw-Hill, New York (1985).

Borra E.F. and Landstreet J.D., "The magnetic fields of the Ap stars”, ApJS 42, 421-445 (1980).

Cairns R.A. et al., “A Cyclotron Maser Instability with Application to Space and Laboratory

Plasmas", Phys. Scr. T116, 23 (2005).

Cairns R.A. et al., “Cyclotron Maser Radiation from an Inhomogeneous Plasma”, Phys. Rev. Lett. 101, 215003 (2008).

Chu, K.R., “The electron cyclotron maser”, Rev. Mod. Phys. 76, 489-540 (2004).

Delory G.T. et.al., "Fast observations of electron distributions within AKR source regions.", Geophys. Res. Lett. 25, 2069-2072 (1998).

Denisov G.G. et al., "Gyrotron traveling wave amplifier with a helical interaction waveguide", Phys. Rev. Lett. 81, 5680-5683 (1998).

Ergun, R.E. et.al., "Electron-cyclotron maser driven by charged-particle acceleration from magnetic field-aligned electric fields”, Astrophysical Journal 538, 456-466 (2000).

Gillespie K. M. et al., “3D PiC code simulations for a laboratory experimental investigation of Auroral Kilometric Radiation mechanisms” Plasma Phys. Controlled Fusion 50, 124038 (2008).

Gurnett D.A., “The Earth as a radio source: Terrestrial kilometric radiation”, J. Geophys. Res. 79, 4227 (1974).

Hatzes A.P., "Doppler imaging of the silicon distribution on CU Vir: Evidence for a decentred magnetic dipole?", Mon. Not. R. Astron. Soc. 288, 153-160 (1997).

Katsouleas, T. and Dawson J.M., "Unlimited Electron Acceleration in Laser-Driven Plasma Waves", Phys. Rev. Lett., 51, 392-395 (1983).

Kellett B.J. et al., "Can late-type active stars be explained by a dipole magnetic trap?", Mon. Not. R. Astron. Soc. 329, 102-108 (2002).

Kellett B.J. et al., “CU Virginis - The First Stellar Pulsar” e-print astro-ph/0701214 (2007). 
Kundu M.R. et al., "Spatial Structure of Simple Spiky Bursts at Microwave/Millimeter Wavelengths", ApJ, 547, 1090-1099 (2001).

Louarn, P. et.al., "Trapped electrons as a free energy source for the auroral kilometric radiation", J. Geophys. Res. 95, 5983-5995 (1990).

Louarn P., Le Queau D., “Generation of the auroral kilometric radiation in plasma cavities -II. The cyclotron maser instability in small size sources”, Planet. Space Sci. 44, 211-224 (1996).

Lo K.K. et al., "Observations and modelling of pulsed radio emission from CU Virginis”, Mon. Not. R. Astron. Soc. 421, 3316-3324 (2012).

McClements K. G. et al., "Lower hybrid resonance acceleration of electrons and ions in solar flares and the associated microwave emission”, ApJ, 409, 465-475, 1993.

McConville S.L. et al., "Demonstration of auroral radio emission mechanisms by laboratory experiment”, Plasma Phys. Controlled Fusion 50, 074010 (2008).

Meier D.L. et al., "Magnetohydrodynamic production of relativistic jets", Science 291, 84-92 (2001).

Melrose D.B. and Dulk G.A., "Electron-cyclotron masers as the source of certain solar and stellar radio bursts", ApJ, 259, 844-858 (1982).

Melrose D.B. 1986, "Instabilities in Space and Laboratory Plasmas”, Cambridge University Press, p202.

Melrose D.B., "Radiation from instabilities in space plasmas.”, Astrophys. Space Sci. 264, 401410 (1998).

Melrose D.B., "Coherent emission in astrophysics: a critique”, Astrophys. Space Sci. 264, 391400, (1999).

Menietti D., Burch J.L., ““"Electron conic” signatures observed in the nightside auroral zone and over the polar cap”, J. Geophys. Res. 90, 5345 (1985).

Menietti J.D. et al., "Simultaneous radio and optical observations of auroral structures:

Implications for AKR beaming” J. Geophys. Res. 116, A12219 (2011).

Mesyats G.A., "Vacuum discharge effects in the diodes of high-current electron accelerators",

IEEE Trans. Plasma Sci. 19, 683-689 (1991).

Mutel R.L. et al., "Cluster multispacecraft determination of AKR angular beaming” Geophys. Res. Letters 35, L07104 (2008).

Nemmen R.S. et al., “A Universal Scaling for the Energetics of Relativistic Jets from Black Hole Systems", Science 338, 1445-1448 (2012).

Noer R.J., "Electron field-emission from broad-area electrodes", Appl. Phys. A 28, 1-24 (1982).

O'Sullivan S.P. and Gabuzda D.C., "Magnetic field strength and spectral distribution of six parsecscale active galactic nuclei jets”, Mon. Not. R. Astron. Soc., 400, 26-42 (2009).

Pritchett P.L., Strangeway R.J., "A Simulation Study of Kilometric Radiation Generation Along an Auroral Field Line”, J. Geophys. Res., [Space Physics] 90, 9650-9662 (1985).

Pritchett P.L., "Electron-cyclotron maser instability in relativistic plasmas", Phys. Fluids 29, 29192930 (1986).

Pritchett P.L. et al., "Free energy sources and frequency bandwidth for the auroral kilometric radiation”, J. Geophys. Res., 104, 10317-10326 (1999). 
Pritchett P.L., Strangeway R.J., Ergun R.E., Carlson C.W., "Generation and propagation of cyclotron maser emissions in the finite auroral kilometric radiation source cavity", J. Geophys. Res., 107, 1437 (2002).

Pyper, D.M. et al., "An abrupt decrease in the rotational period of the chemically peculiar magnetic star CU Virginis”, Astron. Astrophys., 339, 822-830 (1998)

Roux A. et al., 1993, "Auroral kilometric radiation sources: In situ and remote observations from Viking.”, J. Geophys. Res. 98, 11657-11670 (1993).

Ronald K. et al., "Radio frequency resonator structure and diagnostic measurements for a laboratory simulation of Auroral Kilometric Radiation”, Phys. Plasmas 15, 056503 (2008).

Ronald K. et al., "Electron beam measurements for a laboratory simulation of auroral kilometric radiation”, Plasma Sources Sci. Technol. 17, 035011 (2008).

Speirs, D.C. et al., "A laboratory experiment to investigate auroral kilometric radiation emission mechanisms", J. Plasma. Phys., 71, 665-674 (2005).

Speirs D.C. et al., "Numerical simulation of auroral cyclotron maser processes", Plasma Phys. Controlled Fusion 50, 074011 (2008).

Speirs D.C. et al., "Numerical investigation of auroral cyclotron maser processes”, Phys. Plasmas 17, 056501 (2010).

Stix T.H., "Waves in Plasmas", American Institute of Physics, New York (1992).

Strangeway R.J. et al., "FAST observations of VLF waves in the auroral zone: Evidence of very low plasma densities", Geophys. Res. Lett. 25, 2065 (1998).

Tarakanov V.L., KARAT Code User Manual, Berkeley Research Associates Inc.,V.A.,USA (1992).

Thejappa G. et al., "Evidence for the Oscillating Two Stream Instability and Spatial Collapse of Langmuir Waves in a Solar Type III Radio Burst”,

Treumann R.A., "The electron-cyclotron maser for astrophysical application”, Astron. Astrophys. Rev. 13, 229-315 (2006).

Trigilio C., Leto P., Leone F., Umana G., Buemi C., "Coherent radio emission from the magnetic chemically peculiar star CU Virginis", Astron. Astrophys. 362, 281-288 (2000).

Twiss R.Q., "Radiation Transfer and the possibility of negative absorption in Radio Astronomy", Australian Journal of Physics, 11, 564-579 (1958).

Vogt S.S., "A magnetic study of spotted UV Ceti flair stars and related late-type dwarfs", ApJ 240, 567-584 (1980).

Vorgul I., Cairns R.A., Bingham R., "Analysis of a cyclotron maser instability in cylindrical geometry", Phys. Plasmas 12, 122903 (2005).

Winglee, R.M., Pritchett, P.L., "The generation of low-frequency electrostatic waves in association with auroral kilometric radiation.”, J. Geophys. Res. 91, 13531-13541 (1986).

Wu C.S., Lee L.C., “A theory of the terrestrial kilometric radiation”, ApJ. 230, 621-626 (1979).

$\mathrm{Xu}$ N.S. and Latham R.V., "The application of an energy-selective imaging technique to a study of field-induced hot electrons from broad area high voltage electrodes”, Surf. Sci. 274, 147-160 (1992). 
Zarka P., "The auroral radio emissions from planetary magnetospheres - What do we know, what don't we know, what do we learn from them?", Adv. Space Res. 12, 99-115 (1992).

\section{Figure Legends / Captions}

FIG. 1. Diagrammatic representation of the terrestrial auroral process.

FIG. 2. Illustrative diagram showing the formation of a horseshoe shaped velocity distribution in an electron beam subject to magnetic compression.

FIG. 3. Diagrammatic representation of radiation emission from the flare star UVCeti [3].

FIG. 4. Spatial growth rate $\operatorname{Im} \mathrm{k}_{\perp}=\gamma_{\perp}$ normalized to $\Omega_{\mathrm{e} 0} / \mathrm{c}$ as a function of frequency.

FIG. 5. Diagramatic representation of the PiC simulation geometry, with electromagnetically absorbent boundaries representative of free space.

FIG. 6. PiC particle velocity distributions measured on transverse planes within the simulation geometry at (a) $\mathrm{z}=0.36 \lambda_{\text {ce }}$ (b) $\mathrm{z}=11.7 \lambda_{\text {ce }}$ and (c) $\mathrm{z}=18 \lambda_{\text {ce. }}$.

FIG. 7. (a) Temporal evolution of the radial Poynting flux measured in a plane at $r$ $=0.32 \lambda_{\text {ce }}$ spanning the length of the simulation. (b) Fourier transform of $E_{\text {theta }}$ from $\mathrm{t} / \mathrm{t}_{\mathrm{ce}}=0 \rightarrow 540$ at $\mathrm{z}=17 \lambda_{\text {ce }}$.

FIG. 8. 3D contour plot of $\mathrm{E}_{\text {theta }}$ within the simulation geometry. RF emission is evident centred around an axial position of $18 \lambda_{\mathrm{ce}}$ and appears to have a slight backward wave character.

FIG. 9. Schematic diagram of the experimental setup highlighting the magnetic coil configuration and convergent axial magnetic field with peak-plateau region for cyclotron resonant energy transfer. 
FIG. 10. Experimental measurements for the $4.42 \mathrm{GHz}$ resonance, illustrating (a) the spectrum of the output signal, displaying a strong resonance close to the electron cyclotron frequency, $4.42 \mathrm{GHz}$ and (b) the antenna pattern confirming the excitation of the near cut-off $\mathrm{TE}_{0,1}$ mode and yielding the efficiency by integration.

FIG. 11. Experimental measurements for the $11.7 \mathrm{GHz}$ resonance, illustrating (a) the spectrum of the output signal, displaying a strong resonance close to the electron cyclotron frequency, $11.7 \mathrm{GHz}$ and (b) the antenna patterns confirming the excitation of near cut-off modes and yielding the efficiency by integration. 